\title{
Exotic Physics Searches with the ATLAS Detector
}

\author{
James Frost $^{a}$, (On behalf of the ATLAS Collaboration) \\ Department of Physics, Cavendish Laboratory, JJ Thomson Avenue, Cambridge, \\ CB3 OHE, UK \\ Abstract. A selection of the most recent ATLAS results of searches for new physics \\ phenomena are detailed. The proton-proton collision data were collected up to the \\ first half of 2011 with the ATLAS detector at $7 \mathrm{TeV}$ centre-of-mass energy pro- \\ duced by the LHC. Summaries are given of leptonic search signatures, of dilepton \\ resonances, and of a charged lepton and neutrino, interpreted in the context of \\ heavy gauge bosons and Randall-Sundrum gravitons. A search for monojet final \\ states is detailed, and limits placed on large extra-dimensional models. The most \\ recent ATLAS results for dijet resonances are described. Two other search results \\ are outlined: a study of the dijet invariant mass in events with an associated W \\ boson, and a search for first or second generation scalar leptoquarks.
}

\section{Search for Dilepton Resonances}

Several extensions of the Standard Model predict narrow high-mass resonances, which decay into $e^{+} e^{-}$or $\mu^{+} \mu^{-}$pairs. Such models include new heavy spin1 neutral gauge bosons, such as Sequential Standard Model $Z^{\prime}[1]$ or excited boson $Z^{*}[2]$, in adition to spin-2 Randall-Sundrum gravitons, $G^{*}[3]$. The ATLAS detector has been used to search for such resonances in the dilepton invariant mass spectrum [4]. Integrated luminosities of $1.08 \mathrm{fb}^{-1}$ and 1.21 $\mathrm{fb}^{-1}$ of proton-proton collision data has been used for the $e^{+} e^{-}$and $\mu^{+} \mu^{-}$ channels respectively. The predominant Standard Model background is the Drell-Yan process, $Z / \gamma^{*}$, resulting in the same final state. No deviations from Standard Model expectations are observed; consequently limits are set on the cross section times branching fraction, $\sigma B$. The resulting combined limits at 95\% C.L. using a Bayesian approach are $1.83 \mathrm{TeV}$ for the Sequential Standard Model $Z^{\prime}$ boson, and in the range $0.71-1.63 \mathrm{TeV}$ for a Randall-Sundrum graviton with couplings, $k / \bar{M}_{P l}$, in the range $0.01-0.1$. These $Z^{\prime}$ boson limits are the most stringent set to date.

\section{Search for a Heavy Neutral Particle decaying into an Electron- Muon Pair}

The ATLAS Collaboration has searched for neutral new particles which decay into two leptons of differing flavour and opposite charge [5]. Examples include sneutrinos in R-parity violating supersymmetric theories and new gauge bosons $\left(Z^{\prime}\right)$ with lepton-flavour violating interactions. The $e \mu$ final state provides a clean detector signature, with little Standard Model background in the high $m_{e \mu}$ signal region. A luminosity of $0.87 \mathrm{fb}^{-1}$ of $7 \mathrm{TeV}$ collision data is analysed

${ }^{a}$ e-mail: frost@hep.phy.cam.ac.uk 
and no deviations from Standard Model predictions are observed. At 95\% C.L., an upper limit, $\sigma B\left(Z^{\prime} \rightarrow e \mu\right)<11 \mathrm{fb}$ is set for $Z^{\prime}$ masses above $700 \mathrm{GeV}$. Exclusion limits are also placed on the production cross section and R-parity violating couplings for $\tau$-sneutrinos.

\section{Search for a Heavy Gauge Boson decaying into a Charged Lepton and a Neutrino}

Extensions to the Standard Models predicting new heavy gauge bosons [6] can also be investigated through the decay signature of the new charged bosons $\left(W^{\prime}\right)$ to a charged lepton, $e$ or $\mu$, and a neutrino. The ATLAS Collaboration has recently completed such a search, using $1.04 \mathrm{fb}^{-1}$ of $7 \mathrm{TeV} p p$ collision data [7]. The discriminating variable used is the transverse mass, $m_{T}=\sqrt{2 p_{T} E_{T}^{\text {miss }}\left(1-\cos \phi_{l \nu}\right)}$, with a signal manifest as a Jacobian peak lying at high mass on the irreducible background formed by the tail of the Standard Model $\mathrm{W}$ boson decay to the same final state. In the absence of an excess, $95 \%$ C.L. limits are placed upon the cross section times branching fraction; Sequential Standard Model W' bosons with masses up to $2.15 \mathrm{TeV}$ are excluded. These are the best available limits for $m_{W^{\prime}}>600 \mathrm{GeV}$.

\section{Searches in Dijet Mass Distributions}

Dijet mass distributions have shown themselves to be useful tools for investigating new phenomena, and for searching for resonances indicative of new physics. The most recent ATLAS results [8] are obtained from the analysis of $0.81 \mathrm{fb}^{-1}$ of $2011 p p$ collision data. Data events with dijet masses up to $4 \mathrm{TeV}$ are observed, and no evidence of resonant production is found. Limits are set for several new physics scenarios: excluded at 95\% C.L. are excited quarks with masses below $2.91 \mathrm{TeV}$, axigluon masses up to $3.21 \mathrm{TeV}$ and color octet scalar resonances lighter than $1.91 \mathrm{TeV}$.

\section{Searches in Monojet plus Missing Transverse Momentum Final States}

The ATLAS experiment has recently reported a search for new physics in the monojet plus large missing transverse energy final state, which has been updated to a 2011 data sample corresponding to an integrated luminosity of $1.00 \mathrm{fb}^{-1}[9]$. The dominant Standard Model backgrounds, are electroweak gauge boson decays to neutrinos. Model-independent 95\% C.L. upper limits are set on the fiducial cross section for the non-Standard Model production of these final states, varying between $2.02 \mathrm{pb}$ and $0.045 \mathrm{pb}$. Additionally, an 
interpretation is made in terms of the Large Extra Dimensions model [10]. Values of the fundamental $(4+\mathrm{n})$-dimensional Planck scale, $M_{D}$, between 3.2 and $2.0 \mathrm{TeV}$ are excluded for $2-6$ extra dimensions.

\section{Invariant Mass Distribution of Jet Pairs Produced in Association with a Leptonically Decaying W Boson}

ATLAS has performed a study of the invariant mass of jet pairs produced in association with a $W$ boson which decays leptonically, using $1.02 \mathrm{fb}^{-1}$ of 2011 collision data [11]. The CDF Collaboration has published a study of such events, using a $4.3 \mathrm{fb}^{-1}$ sample of TeVatron data, and observes an excess of events in the $120-160$ dijet mass range [12]. The D0 Collaboration find no evidence for such an effect in an equivalent data sample [13]. Both electron and muon channels are analysed, and no significant excess over Standard Model expectations is observed at any mass in the $100-300 \mathrm{GeV}$ search region.

\section{Search for First or Second Generation Leptoquarks}

The ATLAS Collaboration has recently published a search for the pair production of first or second generation scalar leptoquarks using $35 \mathrm{fb}^{-1}$ of $2010 \mathrm{pp}$ collision data [14]. The data are in good agreement with the Standard Model expectation, with no evidence of an excess. Limits at 95\% C.L. are set in the 2 -D plane of the leptoquark mass and the branching fraction, $\beta$, for leptoquark decay into a charged lepton and quark. Lower bounds are set on first and second generation leptoquark masses of $376 \mathrm{GeV}$ and $422 \mathrm{GeV}$ for $\beta=1$. For $\beta=0.5$, the corresponding bounds are $319 \mathrm{GeV}$ and $362 \mathrm{GeV}$.

\section{Summary}

The Exotic physics programme of the ATLAS Collaboration has searched for a wide range of new physics phenomena; Figure 1 summarises a subset of these limits, many of which are world-leading and in excess of $1 \mathrm{TeV}$. No evidence of beyond Standard Model physics has been observed, however the increasing luminosity is opening up new model phase space for searches rapidly. With the superb performance of the LHC during 2011, an end-of-year dataset with at least four times the luminosity of these searches is anticipated.

\section{References}

[1] P. Langacker, arXiv:0911.4294 [hep-ph].

[2] M. Chizhov et al., Physics of Atomic Nuclei 71, 2096 (2008).

[3] L. Randall and R. Sundrum, Phys. Rev. Lett. 833370 (1999). 


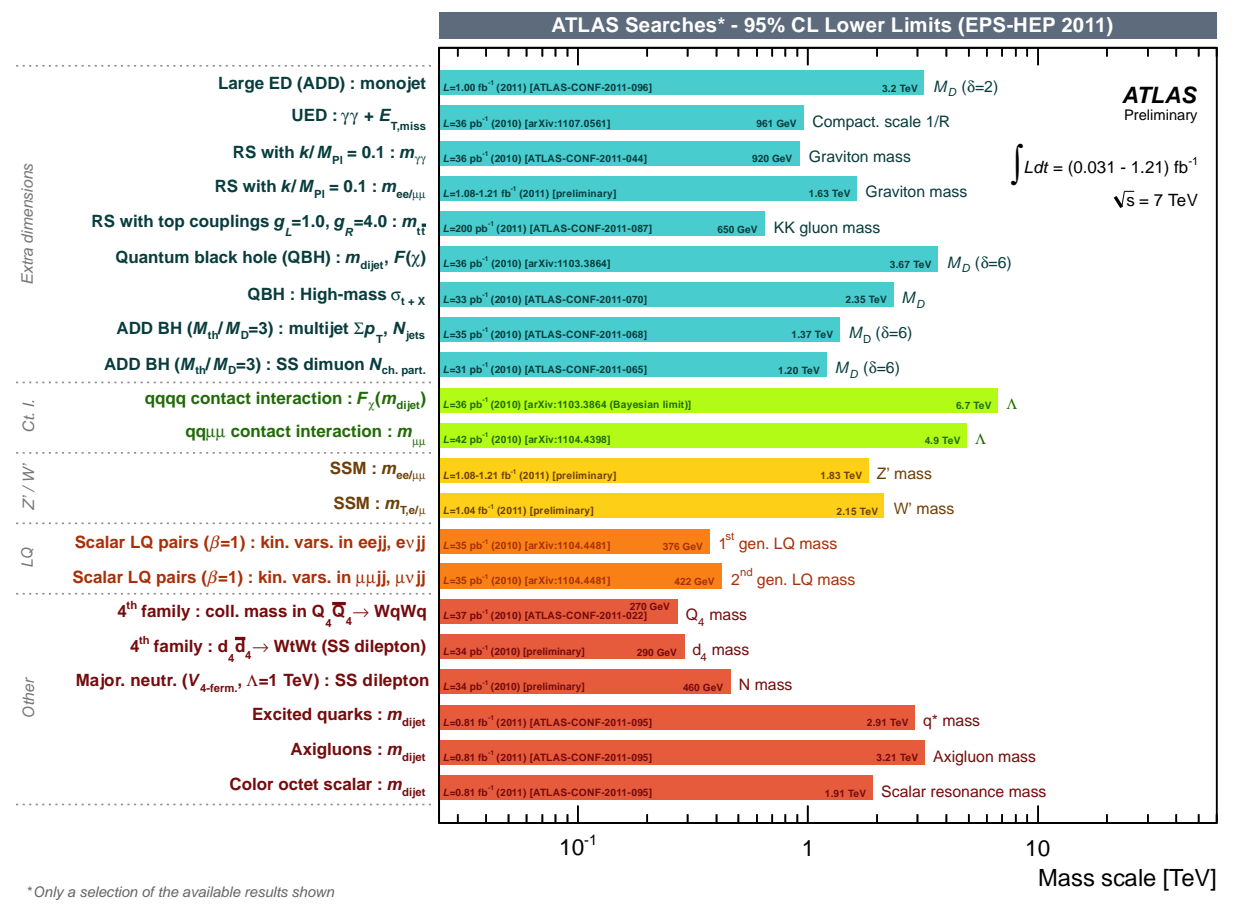

Figure 1: The mass reach of ATLAS Exotic searches for new phenomena (excluding SUSY) - status as of the EPS 2011 conference, 23rd July 2011.

[4] ATLAS Collaboration, accepted by Phys. Rev. Lett., arXiv:1108.1582 [hep-ex].

[5] ATLAS Collaboration, ATLAS-CONF-2011-109, http://cdsweb.cern.ch/record/1373411.

[6] K. Nakamura, et al., J. Phys. G 37075021 (2010).

[7] ATLAS Collaboration, Phys. Lett. B 705 (2011) 28.

[8] ATLAS Collaboration, ATLAS-CONF-2011-095, http://cdsweb.cern.ch/record/1369186.

[9] ATLAS Collaboration, ATLAS-CONF-2011-096, http://cdsweb.cern.ch/record/1369187.

[10] N. Arkani-Hamed, et al., Phys. Lett. B 429263 (1998).

[11] ATLAS Collaboration, ATLAS-CONF-2011-097, http://cdsweb.cern.ch/record/1369206.

[12] CDF Collaboration, Phys. Rev. Lett. 106 (2011) 171801.

[13] D0 Collaboration, arXiv:1106.1921 [hep-ex].

[14] ATLAS Collaboration, Phys. Rev. D 84 011101(R) (2011). 